\title{
IN-GAME BETTING AND THE KELLY CRITERION
}

\author{
ROBIN ANDERSEN, VEGARD HASSEL, LARS MAGNUS HVATTUM \\ AND MAGNUS STÅLHANE
}

\begin{abstract}
When a bet with a positive expected return is available, the Kelly criterion can be used to determine the fraction of wealth to wager so as to maximize the expected logarithmic return on investment. Several variants of the Kelly criterion have been developed and used by investors and bettors to maximize their performance in inefficient markets. This paper addresses a situation that has not, hitherto, been discussed in academic literature: when multiple bets can be placed on the same object and the available odds, true probabilities, or both, vary over time. Such objects are frequently available in sports betting markets, for example, in the case of in-game betting on outcomes of soccer matches. We adapt the Kelly criterion to support decisions in such live betting scenarios, and provide numerical examples of how optimal bet sizes can sometimes be counter-intuitive.
\end{abstract}

\section{INTRODUCTION}

The task of choosing the optimal wealth allocation amongst a set of candidate securities in financial markets is usually referred to as portfolio optimization. This term was first discussed by Markowitz [21] in a paper considered to be the foundation of modern portfolio theory. The core of modern portfolio theory is the mean-variance model, which rests on the assumption that an optimal portfolio can be constructed in such a way that the financial return is maximized for a given risk level or viceversa. The portfolio return is here defined as a linear combination of the returns on the individual investments and the risk coincides with the covariance matrix of these returns.

Another approach to portfolio optimization was proposed by Kelly [15] based on a problem in information theory. The approach is to allocate fractions of wealth to each asset such that these fractions maximize the expected logarithmic growth rate of the investor's wealth. In fact, this allocation also maximizes the expected utility for investors with a logarithmic utility function with respect to their wealth $[4,24]$. This allocation is known as the Kelly criterion and does not necessarily lie on the efficient frontier of the mean-variance model [32].

The Kelly criterion has become a popular tool in betting [16] as well as a supplement for academic research on inefficiencies in betting markets. This paper is motivated by the evaluation of odds when betting on the final outcome of a soccer

$M S C$ (2010): primary 91B06, 91B16; secondary 91.

Keywords: gambling, sport, probability, utility, live odds.

The authors wish to thank an anonymous reviewer for providing comments that helped us to significantly improve an initial version of this manuscript. 
match. In particular, we are interested in the evaluation of odds available during the match, for in-game betting. In this setting, both available odds and true probabilities may fluctuate widely over time when considering the same match. The Kelly criterion was initially developed for binary outcomes: either a bet is won or it is lost. It was later generalized for situations where a single object has multiple mutually exclusive outcomes, and bets can be placed on several of the outcomes.

For the application motivating this work, the additional factor is that odds and true probabilities change over time, and at a given point in time the bettor may need to take into account previously placed bets when deciding how to size any additional bets. To deal with this situation, we develop a mathematical optimization model that can be used to determine optimal bet sizes for in-game betting. The model is shown to be convex so that it can easily be solved using standard software such as Excel Solver. We provide numerical examples showing how the model improves on versions of the Kelly criterion that ignores past bets on the same match, and showing how the optimal decisions are sometimes counter-intuitive.

The rest of this paper is split into five sections. Section 2 provides details of the standard Kelly criterion for the situation with two possible outcomes. Relevant literature is reviewed in Section 3. Section 4 first outlines a model of Kelly for mutually exclusive outcomes and then presents a generalization of this model suitable for in-game betting. Illustrative numerical examples are shown in Section 5, followed by concluding remarks in Section 6 .

\section{The Kelly CRITERION}

The Kelly criterion can be used to determine an optimal bet size for a repeated bet with only two possible outcomes: either the bet is won, returning a profit of the net odds $b$ times the bet size $f$, or the bet is lost, implying a loss of wealth corresponding to $f$. With this definition of the net odds $b$, the decimal odds is equal to $d=b+1$. For fair odds, that is, when a wager has an expected value of 0 , the true probability is therefore $p=1 / d=1 /(b+1)$.

Following [23], let $\boldsymbol{Y}=\left(Y_{i}\right)_{i=1}^{N}$ denote a sequence of i.i.d. random variables for event $i \in\{1, \ldots, N\}$, with $Y_{i} \in\{0,1\}$. Let $p$ be the known probability of the reference outcome $Y_{i}=1$. Now, assume that a bettor successively bets a fixed proportion $f \in[0,1]$ of its initial wealth $W_{0}$ on the events with net odds $b$. Leveraging is not allowed, as implied by $f \leq 1$. The compounded wealth after wagering the same fraction on all $N$ events is then given by

$$
W_{N}(f, \boldsymbol{Y})=W_{0}(1+f b)^{\sum_{i=1}^{N} Y_{i}}(1-f)^{N-\sum_{i=1}^{N} Y_{i}} .
$$

The asymptotic logarithmic return of the fixed fractional betting scheme can then be defined as

$$
G(f)=\lim _{N \rightarrow \infty} \frac{1}{N} \ln \left(\frac{W_{N}(f, \boldsymbol{Y})}{W_{0}}\right),
$$

which, by substitution from Equation (2.1), becomes

$$
G(f)=\lim _{N \rightarrow \infty} \frac{1}{N} \ln \left((1+f b)^{\sum_{i=1}^{N} Y_{i}}(1-f)^{N-\sum_{i=1}^{N} Y_{i}}\right) .
$$

Now, the derivation of the Kelly criterion rests on the following result [17]: 
Theorem 2.1. (Strong Law of Large Numbers) Suppose $X_{1}, \ldots, X_{N}$ are i.i.d. random variables, that $\mathbb{E}\left[X_{i}\right]=\mu$, and that $\mathbb{E}\left[\left|X_{i}\right|\right]$ is finite. Define $\bar{X}_{N}=$ $\frac{1}{N} \sum_{i=1}^{N} X_{i}$. Then, $\bar{X}_{N}$ converges almost surely to $\mu$, that is

$$
P\left(\lim _{N \rightarrow \infty} \bar{X}_{N}=\mu\right)=1 \text {. }
$$

Since the random variables in Equation $(2.1)$ are given by $X_{i}=(1+f b)^{Y_{i}}(1-$ $f)^{1-Y_{i}}$ with corresponding expected value $\mathbb{E}\left[\left|X_{i}\right|\right]=p(1+f b)+(1-p)(1-f) \in$ $[1-f, 1+f b]$, Equation (2.2) converges almost surely to

$$
G(f)=\ln \left((1+f b)^{p}(1-f)^{1-p}\right)
$$

by [15, Thm. 2.1]. Maximization of this function with respect to $f$ provides the following rule:

$$
f^{*}= \begin{cases}\frac{p(b+1)-1}{b}, & \frac{p(b+1)-1}{b}>0, \\ 0, & \frac{p(b+1)-1}{b} \leq 0 .\end{cases}
$$

This is the Kelly criterion for the stated scenario. If short positions $f<0$ were allowed, one would bet $\frac{1-p(b+1)}{b}$ in the case where this quantity is positive [23].

\section{Extant Literature}

This section presents existing research related to betting strategies in sports. The emphasis is placed on the Kelly criterion based on the assumptions that bettors act according to a logarithmic utility function. Conditional returns are fixed by the odds in these markets, a property not shared by most financial markets. Due to this considerable difference in nature, research considering applications of the Kelly criterion in markets with uncertain conditional returns [35] or an unknown probability distribution [31] is omitted.

The Kelly criterion is widely used among bettors in high-frequency games, as the optimal logarithmic growth property of this strategy only holds in the limit $N \rightarrow \infty$ for the number of placed bets $N$ [29]. The Kelly criterion presented in Section 2 rests on the assumptions that the events corresponding to successive bets are i.i.d. binary random variables and that the bettor has perfect information about the probabilities in each event. The former assumption is very restrictive and does not guarantee optimal betting on several mutually exclusive events such as the outcome of a soccer match, which is either a home win, a draw, or an away win. Furthermore, each side of a betting contract only holds estimates of the true probabilities of the outcomes. Thus, some adjustments to the Kelly criterion have been proposed for portfolio optimization in a sports betting market, some of which are presented in the following.

MacLean et al. [19] presented a thorough examination of the existing literature on the properties of the Kelly criterion. An important property is that the Kelly criterion is an optimal myopic strategy, meaning that the strategy is constant regardless of prior and subsequent bets under the presented assumptions. Hakansson [11] proved that this property extends to investments on dependent events given the logarithmic utility function, while Algoet and Cover [1] showed that past outcomes can be accounted for by maximizing the expected logarithmic 
return conditional on these outcomes. However, none of these papers considered the task of allowing multiple bets at different times on a single outcome of a single event.

The main disadvantage with the Kelly criterion is that its suggested wagers are consistently larger than those implied by rational behaviour according to a logarithmic utility function for short investment horizons [19]. The reason is that the mean is much more important for determining the optimal fractions than the variance [14]. This was also indicated by Hsieh et al. [12], who considered the optimal frequency for updating the Kelly fractions in the case where the sequence of games corresponds to i.i.d. random variables. They suggested that, in the absence of transaction costs, the highest possible frequency is optimal. Another issue was presented by Griffin [10], who suggested that, since the Kelly fractions are fixed as a function of total wealth, the Kelly criterion may yield a lower return than expected. This is because the unweighted geometric return rate converges to half the arithmetic return rate.

The Kelly criterion only holds when there exists perfect knowledge of the winning probability $p$. This has motivated a considerable amount of research conducted on the topic of partial Kelly strategies, which imply shrinking the Kelly fractions $f^{*}$ to $\gamma f^{*}$ where $\gamma \in(0,1)$. MacLean et al. [20] considered the use of these strategies for analysis of dynamic portfolio optimization in discrete time, while Thorp [33] tested the strategies for sports betting as well as for Blackjack and in the stock market. Furthermore, Kadane [13] showed that half-Kelly strategies, which simply means choosing $\gamma=\frac{1}{2}$, do not optimize any utility function exactly, but that partial Kelly strategies approximately maximize the constant relative risk aversion utility function $U(f)=\frac{1-f^{1-w}}{w-1}$ by choosing $f=f^{*}$ and $w=\gamma$. Baker and MacHale [3] examined the Kelly criterion with shrinkage when $p$ is uncertain. They showed that the optimal $\gamma$ is a monotonically decreasing function of the variance in the estimates of $p$. Yet another approach to account for uncertainty in $p$ is presented by $\mathrm{Wu}$ et al. [34], who suggested to use the historical winning rate $\hat{p}_{N}=\frac{\text { number of wins }}{N}$ as the estimate of $p$ after $N$ bets in the betting sequences. They showed that this approach yields similar returns to the perfect information scenario for sufficiently large $N$.

The extension of the Kelly criterion to multivariate portfolios is also a topic often considered in existing research. Nekrasov [25] presented a Kelly strategy for multivariate portfolios in the stock market based on estimates of the first and second order moments of excess returns. Cao et al. [5] followed a similar approach, but also proposed a partial Kelly strategy based on volatility regulation to account for uncertainty. The performance of these approaches relies to a large extent on an accurate estimate of the correlation matrix of the assets in question. Several others considered the use of the Kelly criterion for betting on sports events with more than two mutually exclusive outcomes. O'Shaughnessy [28] derived an adjusted Kelly criterion for betting on outcomes (home win, draw, or away win) of soccer matches on a betting exchange. This criterion accounts for taxes to be paid in the case of a winning bet and the possibility of engaging in short positions. In a similar manner, Noon [27] proposed a strategy for sports betting markets where outcomes are mutually exclusive and where both short and long positions are 
allowed, showing that this strategy maximizes the logarithmic utility. Chapman [6] evaluated the use of the Kelly criterion for spread betting, that is, distributing bets over a range of outcomes for a continuous random variable. This topic was also considered by Fitt [8] for the time of arrival of events in soccer matches, albeit using the approach suggested by Markowitz [21] rather than the Kelly criterion. Smoczynski and Tomkins [30] developed an algorithm based on the Kelly criterion for placing bets on the winner of horse races and proved that it asymptotically maximizes the logarithmic return rate. However, all the mentioned approaches only consider pre-game betting.

\section{DERIVING AN IN-GAME KELLY CRITERION}

In the following, a model to find optimal bets for in-game betting is derived. The model can be used in popular fixed odds live betting markets, such as the outcome of a soccer match. The main characteristics of this situation is that there are several mutually exclusive outcomes, each with their own odds, and that a bettor may have placed several bets on the same object in the past, which may influence the optimal bet sizing for the current time being.

A model for betting on mutually exclusive outcomes is presented in Section 4.1. Then, this is generalized for in-game betting in Section 4.2. The initial model for mutually exclusive outcomes is based on the work by Smoczynski and Tomkins [30].

\subsection{Kelly for mutually exclusive outcomes}

Smoczynski and Tomkins [30] proposed an algorithm for optimal wealth allocation in a static betting scenario when the outcomes are mutually exclusive and no events occur simultaneously. This approach is adapted here so that it can be generalized to in-game betting scenarios. First, some assumptions are stated. A fundamental requirement for the Kelly criterion to be optimal for a bettor is that the bettor acts rationally and according to a logarithmic utility function with respect to its wealth (A1). Furthermore, the Kelly criterion only maximizes the logarithmic growth rate asymptotically. Thus, assume in the following that for any given match $m$, a bettor encounters a sufficiently large amount of matches $N_{m}$ with i.i.d. outcomes to that of $m$ (A2). In addition, assume that this holds for every time instant $t$ during any match (A3). Assume further that the bettor can only bet on mutually exclusive outcomes in every match (A4), that there are no transaction costs (A5), and that there exists no lower limit on bet sizes (A6). Also, suppose that the bettor has perfect information regarding the probabilities (A7).

Formally, take the perspective of a bettor considering the optimal wealth allocation on a given match. Then, by otherwise following the notation in Section 2, let $\Omega_{Y}$ denote the set of possible outcomes for i.i.d. $Y_{i}, i \in\{1, \ldots, N\}$, and let $p_{k} \in(0,1)$ be the known probability of outcome $k \in \Omega_{Y}$. Now, assume that a bettor successively makes a wager on a subset of the supplied net odds $\boldsymbol{b}=\left\{b_{k}\right\}_{k \in \Omega_{Y}}$, with $b_{k} \in(0, \infty)$, which is fixed over all these events, by placing a fixed proportion $\boldsymbol{f}=\left(f_{k}\right)_{k \in \Omega_{Y}}$ of their initial wealth $W_{0}$. Furthermore, assume that neither leveraging (A8) nor short positions (A9) are allowed. The two latter assumptions can be formally stated as $\sum_{k \in \Omega_{Y}} f_{k}<1$ and $f_{k} \in[0,1), k \in \Omega_{Y}$, respectively. Then, 
the combined compounded wealth of the bettor after wagering on $N$ matches is given by

$$
W_{N}(\boldsymbol{f}, \boldsymbol{Y})=W_{0} \prod_{k \in \Omega_{Y}}\left(1+f_{k}\left(b_{k}+1\right)-\sum_{j \in \Omega_{Y}} f_{j}\right)^{\sum_{i=1}^{N} I\left(Y_{i}=k\right)},
$$

where $I\left(Y_{i}=k\right)$ is defined as 1 if the outcome of $Y_{i}$ was $k$, and 0 otherwise. The equation follows from the fact that the bettor pays the fraction $\sum_{j \in \Omega_{Y}} f_{j}$ regardless of the outcome of $Y_{i}$, and receives the payment $f_{k}\left(b_{k}+1\right)$ if $y_{i}=k$. Now, this yields an asymptotic logarithmic growth rate

$$
G(\boldsymbol{f})=\lim _{N \rightarrow \infty} \frac{1}{N} \ln \left(\prod_{k \in \Omega_{Y}}\left(1+f_{k}\left(b_{k}+1\right)-\sum_{j \in \Omega_{Y}} f_{j}\right)^{\sum_{i=1}^{N} I\left(Y_{i}=k\right)}\right) .
$$

In a similar manner to the binary equivalent in Equation (2.2), and as a special case of the in-game betting situation considered in the next section, this converges almost surely to

$$
\begin{array}{r}
G(\boldsymbol{f})=\sum_{k \in \Omega_{Y}} \ln \left(\left(1+f_{k}\left(b_{k}+1\right)-\sum_{j \in \Omega_{Y}} f_{j}\right)^{p_{k}}\right)= \\
\sum_{k \in \Omega_{Y}} p_{k} \ln \left(1+f_{k}\left(b_{k}+1\right)-\sum_{j \in \Omega_{Y}} f_{j}\right)
\end{array}
$$

by the strong law of large numbers. The corresponding optimization problem can be formally stated as

$$
\begin{array}{lll}
(P) \max _{\boldsymbol{f}} & \mathrm{G}(\boldsymbol{f}) & \\
\text { subject to } & f_{k} \geq 0, & k \in \Omega_{Y}, \\
& \sum_{k \in \Omega_{Y}} f_{k} \leq 1, & \\
& 1+f_{k}\left(b_{k}+1\right)-\sum_{j \in \Omega_{Y}} f_{j}>0, \quad k \in \Omega_{Y} .
\end{array}
$$

Smoczynski and Tomkins [30] showed that $P$ is a convex optimization problem, and then provided an algorithm that finds the optimum of Equation (4.2).

\subsection{Kelly for in-game betting}

This section presents a strategy that is applicable for the case where subsequent bets can be placed on mutually exclusive outcomes and the return is fixed conditional upon the outcome. The Kelly criterion is an optimal myopic betting strategy given (A1) when at most one bet is allowed for a single outcome of a given event. However, for in-game betting, a bettor is not subject to this restriction. Thus, one should account for the expected return of the previously placed bets in the investment decisions at any given time during the lifetime of the odds. Although the investment decision should ideally incorporate information about the expected path of probabilities until maturity, this is outside the scope of this paper. Thus, 
the bettor is only allowed to take into account the amount already wagered when making this decision (A10).

Now, assume that assumptions (A1)-(A10) hold, and consider a given time $t$ in a hypothetical sequence of $N$ identical matches. By (A2) and (A3), the optimization problem is equal for all these $N$ matches. Thus, the sequence of optimal bets made before time $t$ in all matches are also equal and, by applying (A2) once more, the optimization problem at $t$ is similar in all these matches. In other words, the optimal strategy is myopic across all $N$ matches at a given time $t$.

Before considering the mathematics of this problem, some further notation and restrictions are required. First, let $T=\{1, \ldots, t-1, t\}$, where $t$ is the current time, and $\{1, \ldots, t-1\}$ are the previous moments in time where bets have been placed. The supplied net odds at time $l \in T$ is given as $\boldsymbol{b}_{l}=\left\{b_{k l}\right\}_{k \in \Omega_{Y}}, b_{k l} \in(0, \infty)$, at which point in time $\boldsymbol{f}_{l}=\left(f_{k l}\right)_{k \in \Omega_{Y}}$ of the initial wealth $W_{0}$ is wagered. Then, let

$$
r_{k t}=\sum_{l=0}^{t-1}\left(f_{k l}\left(b_{k l}+1\right)-\sum_{j \in \Omega_{Y}} f_{j l}\right)
$$

denote the fixed return of bets placed at time instants $\{1, \ldots, t-1\}$, where $t$ is the current time instant in a match, conditional on the outcome $y=k$. Furthermore, let

$$
\phi_{t}=\sum_{l=0}^{t-1}\left(\sum_{j \in \Omega_{Y}} f_{j l}\right)
$$

be the total fraction of the bettor's initial wealth $W_{0}$ wagered up until time $t$ during the match. Then, the no leveraging assumption (A8) implies that

$$
\sum_{j \in \Omega_{Y}} f_{j t}<1-\phi_{t}
$$

must hold. Now, consider the optimization problem in the stated scenario. After wagering on $N$ identical matches, the wealth of the bettor is

$$
W_{N, t}(\boldsymbol{f}, \boldsymbol{Y})=W_{0} \prod_{k \in \Omega_{Y}}\left(1+r_{k t}+f_{k t}\left(b_{k t}+1\right)-\sum_{j \in \Omega_{Y}} f_{j t}\right)^{\sum_{i=1}^{N} I\left(Y_{i}=k\right)} .
$$

Then, as in the static scenario, the asymptotic logarithmic growth is

$$
\begin{aligned}
G\left(\boldsymbol{f}_{t}\right)= & \lim _{N \rightarrow \infty} \frac{1}{N} \ln \left(\prod_{k \in \Omega_{Y}}\left(1+r_{k t}+f_{k t}\left(b_{k t}+1\right)-\sum_{j \in \Omega_{Y}} f_{j t}\right)^{\sum_{i=1}^{N} I\left(Y_{i}=k\right)}\right) \\
& :=\lim _{N \rightarrow \infty} \frac{1}{N} G_{N}\left(\boldsymbol{f}_{t}\right)
\end{aligned}
$$

To proceed in the same manner as earlier, it must be shown that Theorem 2.1 applies for Equation (4.3). But first, by the assumption of no allowed short positions (A9), note that

$$
1+r_{k t}+f_{k t}\left(b_{k t}+1\right)-\sum_{j \in \Omega_{Y}} f_{j t}>0, \quad k \in \Omega_{Y},
$$


must hold. In fact, this is necessary to validly state the following:

Proposition 4.1. By Theorem 2.1, $G\left(\boldsymbol{f}_{t}\right)$ converges almost surely to $\sum_{k \in \Omega_{Y}} p_{k t} \ln \left(1+r_{k t}+f_{k t}\left(b_{k t}+1\right)-\sum_{j \in \Omega_{Y}} f_{j t}\right)$.

Proof. Let $X_{i}\left(\boldsymbol{f}_{t}\right)=\ln \left(\prod_{k \in \Omega_{Y}}\left(1+r_{k t}+f_{k t}\left(b_{k t}+1\right)-\sum_{j \in \Omega_{Y}} f_{j t}\right)^{I\left(Y_{i}=k\right)}\right)$. Then, since $\ln \left(a^{\sum_{i=1}^{N} Y_{i}}\right)=\left(\sum_{i=1}^{N} Y_{i}\right) \ln (a)$, we have $G_{N}\left(\boldsymbol{f}_{t}\right)=\sum_{i=1}^{N} X_{i}\left(\boldsymbol{f}_{t}\right)$. Now:

$$
\mathbb{E}\left[\left|X_{i}\left(\boldsymbol{f}_{t}\right)\right|\right]=\left|\sum_{k \in \Omega_{Y}} p_{k t} \ln \left(1+r_{k t}+f_{k t}\left(b_{k t}+1\right)-\sum_{j \in \Omega_{Y}} f_{j t}\right)\right| .
$$

By Equation (4.4), the fact that the odds are finite and positive, and since $p_{k t} \in(0,1), k \in \Omega_{Y}$, while $|\ln (a)|<\infty$ for $a>0$, it follows that $\mathbb{E}\left[\left|X_{i}\left(\boldsymbol{f}_{t}\right)\right|\right]<\infty$. Thus, by Theorem $2.1, \frac{1}{N} G_{N}\left(\boldsymbol{f}_{t}\right)$ converges almost surely to

$$
\sum_{k \in \Omega_{Y}} p_{k t} \ln \left(1+r_{k t}+f_{k t}\left(b_{k t}+1\right)-\sum_{j \in \Omega_{Y}} f_{j t}\right) \text {. }
$$

From this result, the asymptotic logarithmic growth rate is given by

$$
G\left(\boldsymbol{f}_{t}\right)=\sum_{k \in \Omega_{Y}} p_{k t} \ln \left(1+r_{k t}+f_{k t}\left(b_{k t}+1\right)-\sum_{j \in \Omega_{Y}} f_{j t}\right) .
$$

Given the stated assumptions and Equation (4.5), the optimization problem in question can be defined formally as

$$
\begin{array}{rl}
\max _{\boldsymbol{f}_{t}} & G\left(\boldsymbol{f}_{\boldsymbol{t}}\right), \\
\text { subject to } \quad & f_{k t} \geq 0, \\
& \sum_{k \in \Omega_{Y}} f_{k t}<1-\phi_{t}, \\
& 1+r_{k t}+f_{k t}\left(b_{k t}+1\right)-\sum_{j \in \Omega_{Y}} f_{j t}>0, \quad k \in \Omega_{Y},
\end{array}
$$

The stated constraints are labelled as (A), (B), and (C) for later reference. The difficulty of obtaining optimal fractions to wager depends on the complexity of the optimization problem stated above. We intend to show that this is an easy optimization problem, in the sense that it is a convex problem, implying that any locally optimal solution is a global optimum.

First, let us recall some definitions and known results. The definitions of a concave function and a convex set are given by Lundgren et al. [18, pp. 30-31]:

Definition 4.2. A set $X \subseteq \mathbb{R}^{n}$ is a convex set if for any pair of points $\boldsymbol{x}^{(\mathbf{1})}, \boldsymbol{x}^{(\mathbf{2})} \in X$ and $0 \leq \lambda \leq 1$ we have

$$
\boldsymbol{x}=\lambda \boldsymbol{x}^{(1)}+(1-\lambda) \boldsymbol{x}^{(2)} \in X .
$$


Definition 4.3. The function $g(\boldsymbol{x})$ is a concave function on a convex region $X$ if for all choices of points $\boldsymbol{x}^{(1)}, \boldsymbol{x}^{(2)} \in X$ and $0 \leq \lambda \leq 1$ we have that $f\left(\lambda \boldsymbol{x}^{(1)}+\right.$ $\left.(1-\lambda) \boldsymbol{x}^{(2)}\right) \geq \lambda f\left(\boldsymbol{x}^{(1)}\right)+(1-\lambda) f\left(\boldsymbol{x}^{(2)}\right)$.

If $g(\boldsymbol{x})$ satisfies a strict inequality in Definition 4.3 , it is a strictly concave function. Next, if a function $g(\boldsymbol{x})$ is concave, then the function $f(\boldsymbol{x})=-g(\boldsymbol{x})$ is convex [18, p. 30]. Furthermore, the following theorem states that a linear combination of convex functions is a convex function [18, p. 248]:

Theorem 4.4. If $f_{1}(\boldsymbol{x}), f_{2}(\boldsymbol{x}), \ldots, f_{p}(\boldsymbol{x})$ are convex functions and we have $\lambda_{k} \geq 0, k=1, \ldots, p$, then the function $f(\boldsymbol{x})=\sum_{k=1}^{p} \lambda_{k} f_{k}(\boldsymbol{x})$ is convex.

Based on the two latter results, if $f_{k}(\boldsymbol{x})=-g_{k}(\boldsymbol{x})$ for $k \in\{1, . ., K\}$, and $g_{1}(\boldsymbol{x}), \ldots, g_{K}(\boldsymbol{x})$ are all concave and $f(\boldsymbol{x})$ is defined as in Theorem 4.4 , the following holds:

$$
\begin{aligned}
g(\boldsymbol{x}) & =\sum_{k=1}^{p} \lambda_{k}\left(g_{k}(\boldsymbol{x})\right)=\sum_{k=1}^{p} \lambda_{k}\left(-f_{k}(\boldsymbol{x})\right) \\
& =-\sum_{k=1}^{p} \lambda_{k} f_{k}(\boldsymbol{x})=-f(\boldsymbol{x}) \text { concave. }
\end{aligned}
$$

Now, the definition of a convex maximization problem is given by Lundgren et al. [18, p. 29]:

Definition 4.5. A maximization problem $(P)$

$$
\max _{\boldsymbol{x} \in X} g(\boldsymbol{x})
$$

is convex if $X$ is a convex set and $g(\boldsymbol{x})$ is concave on $X$.

If a maximization problem $(P)$ is convex, then each local maximum is also a global maximum. If $g(\boldsymbol{x})$ is strictly concave, then the maximum is also unique $[18$, p. 245]. Lastly, note that the following holds [7, p. 8]:

Theorem 4.6. An intersection of convex sets is itself a convex set.

To show that $(*)$ is convex, it suffices to show that the feasible region $\mathcal{R}^{*}$ is convex and that the objective function $G\left(\boldsymbol{f}_{t}\right)$ is concave on $\mathcal{R}^{*}$ by Definition 4.5 . This is shown below.

Proposition 4.7. The feasible region $\mathcal{R}^{*}$ defined by constraints $(\mathrm{A}),(\mathrm{B})$, and (C) is a convex set.

Proof. First, let $\mathcal{R}^{C}=\left\{\boldsymbol{f}_{t} \in \mathbb{R}^{\left|\Omega_{Y}\right|}: \boldsymbol{f}_{t}\right.$ satisfies $\left.(\mathrm{C})\right\}$. Since $\mathcal{R}^{C}$ is the intersection of the sets $R_{k}^{C}=\left\{f_{k t} \in \mathbb{R}: 1+r_{k t}+f_{k t}\left(b_{k t}+1\right)-\sum_{j \in \Omega_{Y}} f_{j t}>0\right\}$, then it suffices to show that $R_{k}^{C}$ is convex for an arbitrary $k \in \Omega_{Y}$ to validly claim that $\mathcal{R}^{C}$ is convex by Theorem 4.6. Thus, let $f_{k t}^{1}, f_{k t}^{2} \in R_{k}^{C}$ for some arbitrary $k \in \Omega_{Y}$, and let $\lambda \in[0,1]$, where $f_{k t}^{1}, f_{k t}^{2}, \lambda$ is chosen arbitrarily. Then,

$$
\begin{aligned}
& 1+r_{k t}+\left(\lambda f_{k t}^{1}+(1-\lambda) f_{k t}^{2}\right)\left(b_{k t}+1\right)-\lambda \sum_{j \in \Omega_{Y}} f_{j t}^{1}-(1-\lambda) \sum_{j \in \Omega_{Y}} f_{j t}^{2} \\
= & \lambda\left(1+r_{k t}+f_{k t}^{1}\left(b_{k t}+1\right)-\sum_{j \in \Omega_{Y}} f_{j t}^{1}\right)+(1-\lambda)\left(1+r_{k t}+f_{k t}^{2}\left(b_{k t}+1\right)-\sum_{j \in \Omega_{Y}} f_{j t}^{2}\right)
\end{aligned}
$$




$$
>\lambda 0+(1-\lambda) 0=0, \quad \text { so } \quad \lambda f_{k t}^{1}+(1-\lambda) f_{k t}^{2} \in R_{k}^{C} .
$$

Thus, by Definition $4.2, R_{k}^{C}$ is convex, and $\mathcal{R}^{C}=\bigcap_{k \in \Omega_{Y}} R_{k}^{C}$ is convex, since $k$ was chosen arbitrarily. Consider similarly $\mathcal{R}^{B}=\left\{\boldsymbol{f}_{t} \in \mathbb{R}^{\left|\Omega_{Y}\right|}: \boldsymbol{f}_{t}\right.$ satisfies (B) $\}$. Now,

$\lambda \sum_{k \in \Omega_{Y}} f_{k t}^{1}+(1-\lambda) \sum_{k \in \Omega_{Y}} f_{k t}^{2}<\lambda\left(1-\phi_{t}\right)+(1-\lambda)\left(1-\phi_{t}\right)=1-\phi_{t}$, so $\lambda \boldsymbol{f}_{t}^{1}+(1-\lambda) \boldsymbol{f}_{t}^{2} \in \mathcal{R}^{B}$ for any $f_{k t}^{1}, f_{k t}^{2} \in \mathcal{R}^{B}$ and $\lambda \in[0,1]$. Thus, $\mathcal{R}^{B}$ is also convex by Definition 4.2.

Finally, define $\mathcal{R}^{A}=\left\{\boldsymbol{f}_{t} \in \mathbb{R}^{\left|\Omega_{Y}\right|}: \boldsymbol{f}_{t}\right.$ satisfies (A) $\}$. By the same argument, the set of fractions $\mathcal{R}^{A}$ of $\boldsymbol{f}_{t}$ satisfying (A) is also convex since $\lambda \sum_{k \in \Omega_{Y}} f_{k t}^{1}+(1-$ $\lambda) \sum_{k \in \Omega_{Y}} f_{k t}^{2} \geq \lambda 0+(1-\lambda) 0=0$ for any $f_{k t}^{1}, f_{k t}^{2} \in \mathcal{R}^{A}$ and $\lambda \in[0,1]$.

Now, since $\mathcal{R}^{*}$ is the intersection of the convex sets $\mathcal{R}^{A}, \mathcal{R}^{B}, \mathcal{R}^{C}$, then it is also convex by Theorem 4.6 .

Proposition 4.8. The function $G\left(\boldsymbol{f}_{t}\right)$ is convex on $\mathcal{R}^{*}$.

Proof. To show that this proposition holds, define

$$
g_{k}\left(\boldsymbol{f}_{t}\right)=\ln \left(1+r_{k t}+f_{k t}\left(b_{k t}+1\right)-\sum_{j \in \Omega_{Y}} f_{j t}\right) .
$$

Consider $\mathcal{R}^{C}$ defined above. A basis of the proof is that $g_{k}\left(\boldsymbol{f}_{t}\right)$ is concave on $\mathcal{R}^{C}$ for any $k \in \Omega_{Y}$. This follows directly from the fact that $\ln (a)$ is strictly concave on its entire domain $\mathbb{R}_{>0}$, and that $1+r_{k t}+f_{k t}^{1}\left(b_{k t}+1\right)-\sum_{j \in \Omega_{Y}} f_{j t}^{1}>0$ for any $k \in \Omega_{Y}$ and any $\boldsymbol{f}_{t} \in \mathcal{R}^{C}$.

Thus, by Theorem 4.4, $G\left(\boldsymbol{f}_{t}\right)=\sum_{k \in \Omega_{Y}} p_{k t} g_{k}\left(\boldsymbol{f}_{t}\right)$ is also concave on $\mathcal{R}^{C}$, as $p_{k t}>0, k \in \Omega_{Y}$.

Now, it suffices to show that this implies that $G\left(\boldsymbol{f}_{t}\right)$ is concave on $\mathcal{R}^{*}$. Note that since $\mathcal{R}^{*}$ is the intersection of $\mathcal{R}^{A}, \mathcal{R}^{B}, \mathcal{R}^{C}$, then $\mathcal{R}^{*} \subseteq \mathcal{R}^{C}$. Hence, since $\mathcal{R}^{*}$ is itself convex, we have that

$\lambda \boldsymbol{f}_{t}^{1}+(1-\lambda) \boldsymbol{f}_{t}^{2}=\tilde{\boldsymbol{f}}_{t} \in \mathcal{R}^{*}$ for any $\boldsymbol{f}_{t}^{1}, \boldsymbol{f}_{t}^{2} \in \mathcal{R}^{*} \subseteq \mathcal{R}^{C}$ and any $\lambda \in[0,1]$ by Definition 4.2.

But then, since $G\left(\boldsymbol{f}_{t}\right)$ is strictly concave on $\mathcal{R}^{C}$, and $\tilde{\boldsymbol{f}}_{t}=\lambda \boldsymbol{f}_{t}^{1}+(1-\lambda) \boldsymbol{f}_{t}^{2} \in$ $\mathcal{R}^{*} \subseteq \mathcal{R}^{C}$ for any $\boldsymbol{f}_{t}^{1}, \boldsymbol{f}_{t}^{2} \in \mathcal{R}^{*}$ and any $\lambda \in[0,1]$, it is also strictly concave on $\mathcal{R}^{*}$.

As stated, since $(*)$ is a convex problem with $G\left(\boldsymbol{f}_{t}\right)$ strictly convex, then any local optimum of $G\left(\boldsymbol{f}_{t}\right)$ on $\mathcal{R}^{*}$ is also a global optimum. However, to guarantee that a global optimum exists, the Weierstrass theorem [2] states that the objective function must be continuous and that the feasible region must be closed and bounded. To see that the objective function (4.5) is continuous, we note that sums and products of continuous functions are continuous, and that compositions of continuous functions are continuous when defined on the appropriate domains [22]. Since $\ln (x)$ is a continuous function for $x>0$, and since $1+r_{k t}+f_{k t}\left(b_{k t}+1\right)-\sum_{j \in \Omega_{Y}} f_{j t}>0$, it follows that $G\left(\boldsymbol{f}_{\boldsymbol{t}}\right)$ is continuous. To ensure that the feasible region is closed and bounded, the strict inequality restrictions $(<$ and $>$ ) must be replaced by non-strict versions $(\leq$ and $\geq$ ) while shifting the right hand sides by a small value $\epsilon$ : 


$$
\begin{array}{lll}
(* *) \max _{\boldsymbol{f}_{t}} & \mathrm{G}\left(\boldsymbol{f}_{\boldsymbol{t}}\right), & \\
\text { subject to } \quad & f_{k t} \geq 0, & \\
& \sum_{k \in \Omega_{Y}} f_{k t} \leq 1-\phi_{t}-\epsilon, & \\
& 1+r_{k t}+f_{k t}\left(b_{k t}+1\right)-\sum_{j \in \Omega_{Y}} f_{j t} \geq \epsilon, \quad k \in \Omega_{Y} .
\end{array}
$$

The parameter $\epsilon$ simply prevents the investor from exhausting all funds, irrespective of the final outcome of the investments. With this modification, a unique optimal solution exists, and thus, the problem can be solved to optimality in a reasonable time for in-game betting purposes by a suitable optimization algorithm $[26]$.

As a final note, no matches are assumed to be played simultaneously in this approach. The proposed framework can be further extended to account for bets on $E$ simultaneous events given that (A2) and (A3) hold for all such sets of simultaneous events. This can be done by altering Equation (4.1) to

$$
\begin{aligned}
& W_{N, t}^{E}\left(\left(\boldsymbol{f}_{e}, \boldsymbol{Y}_{e}\right)_{e \in \Omega_{e}}\right)= \\
& W_{0} \prod_{e \in \Omega_{e}} \prod_{k \in \Omega_{Y}}\left(1+r_{k t e}+f_{k t e}\left(b_{k t e}+1\right)-\sum_{j \in \Omega_{Y}} f_{j t e}\right)^{\sum_{i=1}^{N} I\left(Y_{i e}=k\right)}
\end{aligned}
$$

where $\Omega_{e}=\{1,2, \ldots, E\}$. However, this approach is not pursued further here.

\section{Illustrative EXAmples}

This section presents some numerical examples intended to show how the in-game Kelly model is necessary when prior bets are already active. The first example also includes the basic Kelly criterion to illustrate that it is not sufficient when dealing with multiple mutually exclusive outcomes. In all the examples, calculations are performed using the software Excel. For the two mathematical models, the inbuilt Solver tool is used to find the optimal solution using the generalized reduced gradient method [9]. For the in-game model, a value of $\epsilon=10^{-6}$ is used.

All the examples are based on betting on the outcome of a soccer match, having the three possible outcomes: home win $(\mathrm{H})$, draw (D), and away win (W). Throughout, the known true probabilities are $p_{H}=0.5, p_{D}=p_{A}=0.25$. To break even, bets on these outcomes require decimal odds of $d_{H}=2.0, d_{D}=d_{A}=4.0$, respectively.

\subsection{Failure of basic Kelly}

If decimal odds are provided as $d_{H}=2.2, d_{D}=d_{A}=3.5$, the true probabilities indicate that betting on a home win is profitable. Using the fact that $b_{k}=d_{k}-1$, the Kelly criterion can be applied to any of the three available bets separately. Since only one of the possible bets is profitable, the basic Kelly and the model for mutually exclusive bets provide the same decision: place a bet equal to $f_{H}=8.3 \%$ of your total wealth on the home win. 
However, if decimal odds are given as $d_{H}=2.2, d_{D}=4.2$ and $d_{A}=3.0$, two of the possible bets are profitable for the bettor. Standard Kelly cannot handle this. If we apply the criterion to each bet separately, violating one of the assumptions of the basic criterion, we find bet sizes of $f_{H}=8.3 \%, f_{D}=1.6 \%$, and $f_{A}=0 \%$. However, this only gives an expected growth rate of $G=0.6 \%$, whereas the model for mutually exclusive bets suggests $f_{H}=13.0 \%, f_{D}=5.6 \%$, and $f_{A}=0 \%$, giving an expected growth rate of $G=0.8 \%$.

\subsection{Failure of model for mutually exclusive outcomes}

Let us assume that the model for mutually exclusive outcomes was used pre-game to place bets in the previous example, leading to optimal bet sizes of $f_{H}=8.3 \%$, $f_{D}=1.6 \%$, and $f_{A}=0 \%$. Assume that some time has passed. In this period, while the true probabilities have not changed, live decimal odds have moved to $d_{H}=2.2, d_{D}=3.0$ and $d_{A}=4.2$. If the model for mutually exclusive outcomes is used again, thereby violating one of its assumptions, it would suggest to place new bets of $f_{H}=13.0 \%, f_{D}=0 \%$, and $f_{A}=5.6 \%$ of the total wealth. This would lead to an expected growth rate of $G=1.8 \%$ according to the in-game model.

The in-game model, however, uses the fact that some bets have already been placed on the same object, and suggests the additional bet sizes $f_{H}=11.0 \%$, $f_{D}=0 \%$, and $f_{A}=11.4 \%$, yielding an expected growth rate of $G=2.4 \%$. This shows that profitable betting opportunities can be exploited to a larger extent when explicitly considering already placed bets on the same betting object.

\subsection{Correcting too large bets}

Consider again the example with $d_{H}=2.2, d_{D}=d_{A}=3.5$. All the three models agree that the optimal bet is to place $f_{H}=8.3 \%$ on a home win, for an expected growth rate of $G=0.4 \%$. Let us assume that the bettor makes a mistake and instead places a double bet equal to $f_{H}=16.7 \%$ on a home win. Such a bet has a growth rate of $G=0 \%$. Using the in-game betting model after placing the wrong bet, it suggests that the bettor can improve the position slightly by placing additional bets with negative expectations. In particular, optimal additional bets of $f_{D}=f_{A}=2.2 \%$ improve the expected growth rate to $0.07 \%$.

\subsection{Correcting bets on wrong odds}

In the same setting as the previous example, let us now consider that the bettor places the correct bet size $f_{H}=8.3 \%$, but accidentally places the bet at an odds of $d_{H}=2.1$ instead of the available $d_{H}=2.2$. This leads to an expected growth rate of $G=0.03 \%$, rather than $G=0.4 \%$. If the odds of $d_{H}=2.2$ is still available, perhaps at a different bookmaker, the in-game model suggests that the situation can be improved by placing an additional bet of $f_{H}=0.3 \%$. This small additional bet would increase the expected growth rate to $G=0.035 \%$.

\subsection{Regular in-game betting}

The standard use of the in-game model is when making bets over time as both true probabilities and odds are moving. As before, let us assume that the game starts with true probabilities $p_{H}=0.5, p_{D}=p_{A}=0.25$ and available odds $d_{H}=2.2$, 
$d_{D}=d_{A}=3.5$, leading to an initial bet of $f_{H}=8.3 \%$ on the home win. Later in the game, the true odds have evolved to $p_{H}=0.8, p_{D}=0.15$, and $p_{A}=0.05$. Fair odds are therefore $d_{H}=1.25, d_{D}=6.7$, and $d_{A}=20$.

Assume that the available odds are still slightly underestimating the probability of a home win: $d_{H}=1.3, d_{D}=5.0$, and $d_{A}=15.0$. The current expected growth rate, given the initial bet, is $G=5.88 \%$. Placing an additional bet on a home win has a positive expected value. However, the in-game model can be used to see that the optimal decision for the bettor is to place no additional bets.

Continuing the example, assume that the true probabilities remain as above, and that the market odds have moved to match the true probabilities, but with a margin for the bookmaker: $d_{H}=1.2, d_{D}=6$, and $d_{A}=18$. In this case, even though there are no value bets available, the in-game model suggests to reduce the risk by placing some small bets on the draw and the away win: $f_{D}=0.8 \%$ and $f_{A}=0.3 \%$. These bets slightly increase the expected growth rate from $G=5.88 \%$ to $G=5.90 \%$.

\section{Concluding Remarks}

This work was motivated by the determination of optimal bet sizes when wagering in live betting markets such as in-game betting on the final outcome of a soccer match. While the Kelly criterion is frequently used to determine bet sizes by gamblers and in academic studies of market inefficiencies, it must be adapted to work in a setting where prior bets have been placed on a given object. To this end, we proposed a mathematical optimization model that is convex and can be easily solved to optimality using standard software.

In several numerical examples, we showed that the model can be a useful tool for bettors. The model can also be used to evaluate market efficiency for in-game betting markets. However, as the model assumes that the bettor is only placing bets on one match object at a time, further extensions are necessary if the same approach is to be used when betting on multiple simultaneous matches.

\section{REFERENCES}

[1] P. H. Algoet and T. M. Cover, Asymptotic optimality and asymptotic equipartition properties of log-optimum investment, The Annals of Probability 16 (1988), 876-898.

[2] J.S. Arora, Introduction to Optimum Design, 4th ed., Academic Press, 2017.

[3] R. Baker and I. G. McHale, Optimal betting under parameter uncertainty: Improving the Kelly criterion, Decision Analysis 10 (2013), 189-199.

[4] R. Bellman and R. Kalaba, On the role of dynamic programming in statistical communication theory, IRE Transactions on Information Theory 3 (1957), 197-203.

[5] R. Cao, Z. Liu, S. Wang and W. Zhou, Multivariate volatility regulated Kelly strategy: A superior choice in low correlated portfolios, Theoretical Economics Letters 7 (2017), 1453-1472.

[6] S. Chapman, The Kelly criterion for spread bets, IMA Journal of Applied Mathematics 72 (2006), 43-51.

[7] J. B. Conway, A Course in Functional Analysis, Springer-Verlag, New York, 1985.

[8] A. D. Fitt, Markowitz portfolio theory for soccer spread betting, IMA Journal of Management Mathematics 20 (2008), 167-184.

[9] D. Fylstra, L. Lasdon, J. Watson and A. Waren, Design and use of the Microsoft Excel Solver, INFORMS Journal on Applied Analytics 28 (1998), 29-55. 
[10] P. A. Griffin, Different measures of win rate for optimal proportional betting, Management Science 30 (1984), 1540-1547.

[11] N. H. Hakansson, On optimal myopic portfolio policies, with and without serial correlation of yields, The Journal of Business 44 (1971), 324-334.

[12] C.-H. Hsieh, B. R. Barmish and J.A. Gubner, At what frequency should the Kelly bettor bet?, 2018 Annual American Control Conference, ACC 2018, Milwaukee, WI, USA, June 27-29, IEEE, 2018, pp. 5485-5490.

[13] J. B. Kadane, Partial-Kelly strategies and expected utility: Small-edge asymptotics, Decision Analysis 8 (2011), 4-9.

[14] J.G. Kallberg and W.T. Ziemba, Mis-specifications in portfolio selection problems, in: G. Bamberg and K. Spremann (eds.), Risk and Capital, Proceedings of the 2nd Summer Workshop on Risk and Capital Held at the University of Ulm, West Germany, June 2024, 1983, Lecture Notes in Economics and Mathematical Systems 227, Springer, Berlin, Heidelberg, 1984, pp. 74-87.

[15] J. Kelly, A new interpretation of information rate, IRE Transactions on Information Theory 2 (1956), 185-189.

[16] B. Li and S.C.H. Hoi, Online portfolio selection: A survey, ACM Computing Surveys 46 (2014), 1-36.

[17] M. Loève, Probability Theory I, Graduate Texts in Mathematics, Springer-Verlag, New York, 1977.

[18] J. Lundgren, M. Rönnqvist and P. Värbrand, Optimization, Studentlitteratur AB, 2010.

[19] L. C. MacLean, E. O. Thorp and W.T. Ziemba, Long-term capital growth: The good and bad properties of the Kelly and fractional Kelly capital growth criteria Quantitative Finance 10 (2011), 681-687.

[20] L. C. MacLean, W. T. Ziemba and G. Blazenko, Growth versus security in dynamic investment analysis, Management Science 38 (1992), 1562-1585.

[21] H. Markowitz, Portfolio selection, Journal of Finance 7 (1952), 77-91.

[22] J. E. Marshden and A. J. Tromba, Vector Calculus, 4th ed., W. H. Freeman and Company, New York, 1996.

[23] S. Moffitt, Gambling for quants, Part 1: A simple fractional betting system, http://dx. doi.org/10.2139/ssrn.2914620 (2017), 19 pp.

[24] J. Mossin, Optimal multiperiod portfolio policies, The Journal of Business 41 (1968), 215229 .

[25] V. Nekrasov, Kelly criterion for multivariate portfolios: A model-free approach, http:// dx.doi.org/10.2139/ssrn. 2259133 (2014), 15 pp.

[26] J. Nocedal and S. J. Wright, Numerical Optimization, Springer Series in Operations Research and Financial Engineering, Springer-Verlag, New York, 1999.

[27] E. Noon, Extending Kelly Staking Strategies to Peer-to-Peer Betting Exchanges, thesis, Imperial College London, 2014.

[28] D. O'Shaughnessy, Optimal exchange betting strategy for win-draw-loss markets, in: A. Bedford and A. J. Schembri (eds.), Proceedings of the Eleventh Australasian Conference on Mathematics \& Computers in Sport, 11M\&CS, September 17-19, 2012, Melbourne, Australia, 2012, pp. 62-66.

[29] Z. Peterson, Kelly's criterion in portfolio optimization: A decoupled problem, Journal of Investment Strategies 7 (2018), 53-76.

[30] P. Smoczynski and D. Tomkins, An explicit solution to the problem of optimizing the allocations of a bettor's wealth when wagering on horse races, The Mathematical Scientist 35 (2010), 10-17.

[31] Q. Sun and S. Boyd, Distributional robust Kelly gambling, arXiv:1812.10371 (2019), 12 pp.

[32] E. O. Thorp, Portfolio choice and the Kelly criterion, in: W. T. Ziemba and R. G. Vickson (eds.), Stochastic Optimization Models in Finance, Academic Press, 1975, pp. 599-619.

[33] E. O. Thorp, The Kelly criterion in blackjack sports betting, and the stock market, in: S. A. Zenios and W.T. Ziemba (eds.), Handbook of Asset and Liability Management, NorthHolland, San Diego, 2008, 385-428.

[34] M.-E. Wu, H.-H. Tsai, R. Tso and C.-Y. Weng, An adaptive Kelly betting strategy for finite repeated games, in: T. T. Zin et al. (eds.): Genetic and Evolutionary Computing, 
Proceedings of the Ninth International Conference on Genetic and Evolutionary Computing, August 26-28, 2015, Yangon, Myanmar - Volume II, Springer, Cham, 2016, pp. 39-46.

[35] W. T. Ziemba, The Stochastic Programming Approach to Asset, Liability, and Wealth Management, Research Foundation of CFA Institute, 2003.

Robin Andersen, Department of Industrial Economics and Technology Management, NTNU, Trondheim, Norway

e-mail: robina@stud.ntnu.no

Vegard Hassel, Department of Industrial Economics and Technology Management, NTNU, Trondheim, Norway

e-mail: vegahas@gmail.com

Lars Magnus Hvattum, Faculty of Logistics, Molde University College, Molde, Norway e-mail: hvattum@himolde.no

Magnus Stålhane, Department of Industrial Economics and Technology Management, NTNU, Trondheim, Norway

e-mail: magnus.staalhane@ntnu.no 
